\title{
Dental Treatment Approach in Cantho University of Medicine and Pharmacy of Vietnam
}

\author{
Minh T. Nguyen ${ }^{1}$, Nhan B. Truong ${ }^{2}$, Thoa C. Nguyen ${ }^{3}$ \\ ${ }^{1}$ Ophthalmo-ORL-Maxillo-Facial-Odontology Hospital of An Giang, Vietnam \\ ${ }^{2}$ Department of Epidemiology, Faculty of Public Health, Can Tho University of Medicine and Pharmacy, Vietnam \\ ${ }^{3}$ Department of Prosthodontics, Faculty of Dentistry, Can Tho University of Medicine and Pharmacy, Vietnam \\ Correspondence e-mail to: daisy_minh@yahoo.com
}

\begin{abstract}
The traditional morphological concept to dental care has shown many drawbacks and is not appropriate in many cases. To counteract these shortcomings, a problem-solving approach has been introduced in dental curriculum of Cantho University of Medicine and Pharmacy (CTUMP), Vietnam. This approach should be reflected in dental practice in CTUMP. Objective: To investigate the problem-solving approach to dental care of CTUMP by patterns of tooth extraction, and tooth rehabilitation. Methods: Cross-sectional data on DMF, dental treatments planned, dental treatments delivered from 1549 dental records of patients aged $\geq 18$ of CTUMP were analyzed. Results: The majority of patients were aged 18-29 (929, 60\%), classified as professional and skilled workers (1112 subjects, $72 \%$ ), lived in urban areas (1156 subjects, $75 \%$ ), and women (932, 60\%). The number of teeth eventually receiving dental treatment was lower than the number of teeth indicated for the treatment. On average, each patient had 2 teeth receiving treatment. Tooth restoration was the most common treatment (1390, 70\%). Molars were the most treated teeth $(842,43 \%)$. Molars showed statistically significant higher chance for restoration and extraction than premolars and anterior teeth (Wilcoxon-signed-ranks test $p \leq 0.017$ ). No statistically significance was found in tooth replacement between premolar and molar regions. The dental treatments aimed to preserve all teeth regardless of dental regions. Tooth replacement may tend to be morphologically based rather than functionally as most prostheses restored the complete dental arch. Conclusions: The approach to dental care in CTUMP tends to be morphologically conservative.
\end{abstract}

Key words: dental treatment plan, oral function, probelm-solving approach, tooth extraction, tooth replacement

\section{INTRODUCTION}

\section{Traditional approach to dental care}

Traditional treatment planning in dentistry focuses on morphology. Complete dental arches are considered indispensable to meet oral function demands. In many cases this approach may lead to overtreatment and the dental resource requirements are neither always affordable nor accessible. The treatment is considered as overtreatment when the treatment is solely based on the professional assessment rather than on the needs of patient. ${ }^{1}$ When the treatment is solely based on the professional assessment of the dentists without regarding the subjective perceived needs and the treatment demands of the patients, the treatment may not fulfill the needs of the patients. ${ }^{2,3}$ Consequently, the patients may neither accept nor adapt to the treatment. In the traditional approach, molar support is considered indispensable for occlusal stability and healthy temporomandibular joints. Consequently, all damaged molars should be restored and artificial teeth should replace all missing molars. In this respect it is worthwhile to note that the so-called restorative repair cycle is mostly related to molars since molars are most susceptible to dental disease. ${ }^{4,5}$ This constitutes complicated and costly treatments on the preservation of molars due to an intensive repair recycle of consecutively restoring "permanent" restorations. 
The cost of complex treatment in order to preserve the complete dental arch places a primary burden on the patient and a widespread recourse to managed care has placed severe limitations on the number of patients that may be able to avail themselves of the treatment. ${ }^{6}$

Because of the limitations mentioned above, the traditional morphological approach shifted to a paradigm that is based on biological and functional considerations. These considerations and underlying assumptions for a healthy or physiologic occlusion, as developed by Ramfjord \& Ashand Mohl et al., reflect this shift clearly: [1] Absence of pathologic manifestations; [2] Satisfactory function (aesthetics, chewing, oral comfort); Mandibular stability; [3] Variability in form and function of the stomathognatic system; [4] Adaptive capacity of the stomathognatic system to changing situations. This physiological and functional approach assumes a variety of forms of the dentition, which is still compatible with healthy occlusion and satisfying oral function. An important implication is that the number of teeth may vary and thus may be less than 28 .

The problem-oriented approach in contemporary dentistry implies that the treatment plan focus on solving dental problems of the patient in practical way in order to maintain a healthy functional stomathognatic system. In a problem-oriented approach, patient's dental problems are formulated from relevant information extracted from the medical and dental history and a thorough oral examination The problem-oriented approach is in line with treatment goals as promoted by the WHO (1992): "the retention throughout life of a functional, aesthetic, natural dentition of not less than 20 teeth and not requiring recourse to prostheses". The WHO goal is substantiated by a recently published systematic review. ${ }^{9}$ In this review it was concluded that although in general masticatory efficiency decreases with the loss of teeth, shortened dental arches (SDAs) comprising nine to 10 pairs of occluding teeth (including the anterior teeth) assures masticatory function, occlusal support, and occlusal and mandibular stability for most people. In subjects with SDAs there is sufficient adaptive capacity to maintain adequate oral function when at least four occlusal units are left, preferable in a symmetrical position. ${ }^{1,10}$ There is no evidence that occlusal instability is associated with moderate types of SDA, i.e. intact anterior and premolar regions. ${ }^{11}$ Moreover, for individuals with SDAs there is no evidence of increased signs and symptoms of temporomandibular disorders over the years. ${ }^{12,13}$ In conclusion, occlusal support from an SDA dentition seems to assure sufficient oral function without discomfort. ${ }^{6,14,15} \mathrm{n}$ the last decades, the concepts on occlusion shifted from 'ideal', characterized by mechanical and morphological concepts to 'normal', based on biological considerations. As a result, the acceptance of morphological and functional variability of the stomatognathic system has gained increasing support. This change has consequences for everyday dental practice. ${ }^{16}$

Dental care in Vietnam according to data from the National Oral Health Survey 2001 showed that the prevalence of caries amongst adults in Vietnam is high. ${ }^{17}$ According to this survey, the numbers of decayed/ missing/filled teeth (DMFT) in subjects aged 45 years and over ranged from 6.09 to 11.66 in different regions of Vietnam. The high numbers of missing teeth (4.45 to 8.59 for those aged 45 years and over) combined with the low numbers of filled teeth (0.02 to 0.36$)$ indicates that extraction was the most common treatment for caries and that restorative treatment was low. This data is inline with the result from a cross-sectional epidemiological study on the oral health status of the adults in Southern Vietnam. ${ }^{18}$ This study showed that the majority of adults of Southern Vietnam presented a reduced dentition. In Vietnam, oral health care is not accessible for everyone. The reasons of this disparity may be the shortage of dental workforce and the lack of finance. The dental workforce is not only insufficient but also distributed unevenly. For the Southern region, the dental workforce is approximately 2000 for 36 million people (on average 1 dental professional for 18.000 people). ${ }^{19}$ Most dental practices are in urban areas, very few rural residents have access to any dental health services. An example of the unequal distribution of dental services: in the urban area the ratio of dentist per population is $1 / 13.000$, while in the rural area this ratio is $1 / 180.000$, of which the number of dentists is zero in 156 rural districts (43\% of a total of 363 ) (data from 2008). ${ }^{20}$ Along with the insufficient dental workforce, the health care budget for the population is very limited. The most recent data indicated a total health expenditure of US $\$ 57.5$ per capita in the year 2007 , which is $7.1 \%$ of GDP. ${ }^{21}$ Of this expenditure, the government provides only $8.7 \%$; the remaining part of the cost is paid by the population. ${ }^{21}$ Due to the limited financial resources, the traditional oral health care strategy following morphological approach in Vietnam is unaffordable for both the population and the government. Consequently, the inaccessibility to dental services and the unaffordability of dental care of the Vietnamese population constitute the constraint in the pursuit of the ultimate goal of primary health care 'for all' that is advocated by WHO. To counteract these shortcomings, the problem-solving approach to dental care has been introduced to Vietnam in 2004 by integrating this concept in dental curriculum of Can Tho University of Medicine and Pharmacy (CTUMP). By now, the problem-solving approach should be reflected in dental treatment plan in the dental school. The present study was conducted to investigate the insights into which level the new concept to dental care is adopted in this institution. Specifically. We aimed to investigate the problem-solving approach to dental care of CTUMP by patterns of tooth extraction, and tooth rehabilitation. 


\section{METHODS}

Cross-sectional study using data from dental records of the patients aged $\geq 18$ of the faculty of dentistry of CTUMP in the period of January to July 2009. Records with incomplete administrative information were excluded from this study.

Sample size:

$\begin{array}{cc}Z^{2} p(1-p) & 1.96^{2} \times 0.22 \times(1-0.22) \\ d^{2} & 0.021^{2}\end{array}=1494.8 \approx 1500$

$Z=1.96(\alpha=0,05)$, confidence interval: $95 \%$.

$\mathrm{p}=0.22(*)$

$\mathrm{d}=0.021$

(*): the proportion tooth extraction in Can Tho hospital (Palenstein et al, 2000). ${ }^{27}$

\section{Data collection}

Data were collected by a $6^{\text {th }}$-year dental student who is the first author. After having the permission of the dean of faculty of dentistry, data from dental records of the patients aged $\geq 18$ from dental clinic of CTUMP in the period of January to July 2009 were included in this study. From 1549 dental records, at the patient level, the following informations were collected for each patient: demographic, socio-economic variables: gender, age, residence (urban or rural), and occupation; motivations of dental visit; periodontal status: gingivitis, periodontitis. At the tooth level, the following informations were collected: dental status: healthy, caries, cervical tooth wear, fracture repairable, large fracture unrepairable, apical infection, root, absent, malposition; restoration: no restored, filling, filling and secondary caries repairable, filling and secondary caries unrepairable, pontics, RPD (removable partial denture), crown; dental treatment planned: filling, endodontics, extraction, orthordontics, RPD, FPD (fixed partial denture) or crown, endodontics + crown, tooth extraction + RPD, tooth extraction + FPD and unknown; dental treatments received: filling, endodontics, extraction, orthordontics, RPD, FPD or crown, endodontics + crown, tooth extraction + RPD, tooth extraction + FPD.

\section{Data analysis}

The third molars were excluded in this analysis. Four groups of occupation (professional and skilled, business and household keeper, retired/unemployed, semi-skilled and unskilled) were classified into two group (group $1=$ professional and skilled, group $2=$ business and household keeper, retired/unemployed, semi-skilled and unskilled).

In term of the dental status of the patients before the treatments, to determine the effects of age, gender, residence and occupation on the distribution of decayed (D), missing (M), and restored (R) teeth, multivariate logistic regression analyses were performed for the whole dentition, anterior, premolar and molar regions separately. For these analyses, the variables were dichotomized using the following cut-off points: decay (decay present; no decay), teeth missing (missing; no missing), restoration (restoration present; no restoration present). Codings for the independent variables in the final models of multivariate logistic regression were: age (numerical variable), gender (male vs females), residence (urban vs rural), occupation (group I: professional and skilled vs group II: non-professional). Three types of dental treatment received from CTUMP were analyzed: tooth extraction, restoration (including fillings, endodontic treatment, crowns), and tooth replacements (pontics and removable partial dentures). To determine the chance between 3 types of dental treatment (extraction $v s$ restoration, extraction $v s$ tooth replacement, restoration $v s$ tooth replacement) in a dental region (anterior, premolar, and molar regions), Friedman test and Wilcoxon signed ranks test were performed (the Bonferroni adjustment was used in Wilcoxon signed ranks test: a new significance level of $0.05 / 3=0.017$ was applied, this means that if the $P$ value is larger than 0.017 then we do not have a statistically significant result). The variables in this test were determined by dividing the number of teeth receiving the respective treatment by the total number of teeth receiving all 3 types of treatment in a dental region. If the results from Friedman test showed statistical significant, the Wilcoxon signed ranks test with Bonferroni adjustment would be performed.

$\operatorname{Ext}(\operatorname{ant} / \mathrm{pre} / \mathrm{mol})=$

Number of teeth extracted (ant / pre / mol)

Number of teeth extracted + number of teeth restored + number of teeth replaced (ant $/ \mathrm{pre} / \mathrm{mol}$ )

$\operatorname{Rest}(\operatorname{ant} / \mathrm{pre} / \mathrm{mol})=$

Number of teeth restored (ant / pre / mol)

Number of teeth extracted + number of teeth restored + number of teeth replaced (ant $/ \mathrm{pre} / \mathrm{mol}$ )

$\operatorname{Repl}(\mathrm{ant} / \mathrm{pre} / \mathrm{mol})=$

Number of teeth restored (ant / pre / mol)

Number of teeth extracted + number of teeth restored + number of teeth replaced (ant $/ \mathrm{pre} / \mathrm{mol}$ )

To determine the chance of receiving a type of dental treatment between dental regions (anterior $v s$ premolar, anterior $v s$ molar, premolar $v s$ molar), Friedman test and Wilcoxon signed ranks test were performed (the Bonferroni adjustment was used in Wilcoxon signed ranks test: a new significance level of $0.05 / 3=0.017$ was applied, this means that if the $p$ value is larger than 0.017 then we do not have a statistically significant 
result). The variables in this test were determined by dividing the number of teeth receiving the respective treatment by the total number of teeth in the complete dental region concerned. If the results from Friedman test showed statistical significant, the Wilcoxon signed ranks test with Bonferroni adjustment would be performed.

Ext.rel $=$

Number of teeth extracted in a dental region

Total number of teeth in the complete dental region concerned*

Rest.rel $=$

Number of teeth restored in a dental region

Total number of teeth in the complete dental region concerned*

Repl.rel $=$

Number of teeth replaced in a dental region

Total number of teeth in the complete dental region concerned*

*: Total number of teeth in the complete anterior region in the upper/lower jaw is 6 , in the whole dentition is 12 ; total number of teeth in the complete premolar region in the upper/lower jaw is 4 , in the whole dentition is 8 ; total number of teeth in the complete molar region in the upper/ lower jaw is 4 , in the whole dentition is 8 .

In the problem-solving approach, the priority of the dental care should be focus on the preservation of the anterior and premolar regions which is the strategic part of the dentition. In contrast, in the traditional approach, the dental treatment is aimed to preserving all teeth regardless the dental region, leading to a complete dental arch. From these analysis, the higher chance for rehabilitation (tooth restored and/or tooth replaced) of anterior and premolar teeth (the strategic part of the dentition) would suggest that the problemsolving approach to dental care is applied, otherwise, no-difference between the chance for rehabilitation of the dental regions or higher chance in molar region would suggest that the dental care is considered as tend to be morphological.

\section{RESULTS}

Demographic and socio-economic characteristics Most patients were in young age group. The proportion of patients aged $18-29$ is $60 \%$. Among the patients in this study, 60\% (932 subjects) were women and 40\% (617 subjects) were men. Most of them lived in urban areas (1156 subjects, 75\%) and were professional and skilled (1112 subjects, 72\%). Most of them visited dental clinic for caries (626 cases, 40\%), 370 (24\%) visited for dental check up, and 140 (9\%) visited for tooth extraction.

\section{Dental status before treatments}

Teeth decayed. At all ages subjects in this sample have on average approximately less than 5 decayed teeth in the whole dentition (Figure 1). The mean number of decayed teeth in the molar region decreases with age while the anterior region and premolar region increase (Figure. 2, 3, 4). The multivariate logistic regression analysis (Table 1) shows that each additional year of age gives a significantly lower chance for having decayed teeth in all dental regions and molar region (OR: 0.98; $p \leq 0.001$ and OR: $0.97 ; p \leq 0.001$ respectively). The influence of gender, residence and occupation on decay was not statistically significant. However, urban patients have lower risk for decay than rural patients.

Teeth missing. After the age of approximately 50 years the mean number of missing teeth increases considerably (Figure. 1, 2, 3, 4). The mean number of missing teeth in the whole dentition is 15 at the age of 90 years. Multivariate logistic regression analysis shows the significant increase of missing teeth with age (Table 1). Every additional year gives a 3.3\% higher chance of missing teeth in all dental regions (OR: 1.033; $p \leq 0.001)$. Professional and skilled patients have lower chance for missing teeth in all dental regions (OR: 0.66; $p \leq 0.001$ ), anterior and premolar regions. The influence of gender and residence on missing teeth could not be demonstrated.

Teeth restored. The mean number of restored teeth is low at all ages in all dental regions (Figure. 1, 2, 3, 4). The multivariate logistic regression analysis shows that each additional year of age gives significantly higher chance of having restored teeth in premolar region (OR: 1.027; $p \leq 0.01$ ) (Table 1). Males have fewer restored teeth than females in anterior region (OR: $0.74 ; p \leq$ 0.05). The influence of residence and occupation for restoration were not statistically significant.

\section{Dental treatment planning and dental treatments delivered by CTUMP}

Among 1549 patients, 157 patients (10\%) received tooth extraction with 245 teeth extracted. Decay is the most prominent reason for tooth extraction (222 teeth, 91\%), the extractions due to periodontal disease and other reasons account for a small proportion (20 teeth $(8 \%)$ and 03 teeth (1\%) respectively).

The number of teeth receiving the treatments were lower than the number of teeth indicated for the treatments in the treatment planning. This trend was showed in three types of treatment in the upper jaw, lower jaw and the whole dentition. Overall, each patient had an average of 2 teeth receiving the treatments. The number of teeth indicated to be extracted (560 teeth) was higher than the number of teeth extracted (245 teeth) (Table 2). Besides, 2604 teeth were indicated 
Table 1. The effects of age, gender, residence and occupation on the distribution decay, missing and restoration in the whole dentition, anterior, premolar and molar regions of 1549 patients before treatment.

\begin{tabular}{|c|c|c|c|c|c|c|c|c|c|}
\hline & \multicolumn{3}{|c|}{ Decay } & \multicolumn{3}{|c|}{ Missing } & \multicolumn{3}{|c|}{ Restoration } \\
\hline & $\overline{\mathrm{OR}}$ & $95 \% \mathrm{CI}$ & $\mathrm{P}$ & $\overline{\mathrm{OR}}$ & $95 \% \mathrm{CI}$ & $\mathrm{P}$ & $\overline{\mathrm{OR}}$ & $95 \% \mathrm{CI}$ & $\mathrm{P}$ \\
\hline \multicolumn{10}{|l|}{ All regions } \\
\hline Age (per year) & 0.98 & 0.97-0.99 & $* * *$ & 1.033 & $1.02-1.04$ & $* * *$ & 1.001 & $0.99-1.01$ & \\
\hline Male $^{a}$ & 0.94 & $0.74-1.19$ & & 1.2 & $0.95-1.52$ & & 0.96 & $0.76-1.2$ & \\
\hline Urban $^{\mathrm{b}}$ & 0.82 & $0.62-1.07$ & & 1.13 & $0.87-1.48$ & & 1.02 & $0.79-1.32$ & \\
\hline Occupational group $\mathrm{I}^{\mathrm{c}}$ & 0.86 & $0.62-1.2$ & & 0.66 & $0.48-0.91$ & $* *$ & 0.88 & $0.64-1.2$ & \\
\hline \multicolumn{10}{|l|}{ Anterior region } \\
\hline Age (per year) & 1.004 & $0.99-1.015$ & & 1.057 & $1.04-1.07$ & $* * *$ & 1.004 & $0.99-1.016$ & \\
\hline Male $^{a}$ & 0.85 & $0.67-1.07$ & & 1.08 & $0.7-1.66$ & & 0.74 & $0.55-0.99$ & $*$ \\
\hline Urban $^{\mathrm{b}}$ & 0.87 & $0.67-1.13$ & & 1.42 & $0.88-2.27$ & & 0.96 & $0.7-1.32$ & \\
\hline Occupational group $\mathrm{I}^{\mathrm{c}}$ & 0.76 & $0.55-1.05$ & & 0.47 & $0.28-0.79$ & $* *$ & 0.7 & $0.5-1.04$ & \\
\hline \multicolumn{10}{|l|}{ Premolar region } \\
\hline Age (per year) & 1.004 & $0.99-1.016$ & & 1.055 & $1.04-1.07$ & $* * *$ & 1.027 & $1.01-1.04$ & $* * *$ \\
\hline Male $^{a}$ & 0.91 & $0.71-1.18$ & & 0.99 & $0.69-1.43$ & & 0.74 & $0.51-1.08$ & \\
\hline Urban $^{\mathrm{b}}$ & 0.85 & $0.65-1.13$ & & 1.003 & $0.68-1.46$ & & 1.16 & $0.76-1.77$ & \\
\hline Occupational group I ${ }^{\mathrm{c}}$ & 0.9 & $0.64-1.27$ & & 0.55 & $0.36-0.85$ & $* *$ & 0.92 & $0.57-1.5$ & \\
\hline \multicolumn{10}{|l|}{ Molar region } \\
\hline Age (per year) & 0.97 & $0.96-0.98$ & $* * *$ & 1.04 & $1.03-1.05$ & $* * *$ & 1.005 & $0.99-1.01$ & \\
\hline Male & 1.01 & $0.81-1.26$ & & 1.08 & $0.84-1.38$ & & 1.07 & $0.84-1.37$ & \\
\hline Urban $^{\mathrm{b}}$ & 0.9 & $0.7-1.16$ & & 1.05 & $0.8-1.38$ & & 1.3 & $0.97-1.73$ & \\
\hline Occupational group $\mathrm{I}^{\mathrm{c}}$ & 1.05 & $0.77-1.44$ & & 0.78 & $0.56-1.08$ & & 0.99 & $0.7-1.41$ & \\
\hline
\end{tabular}

$*=P \leq 0.05 ; * *=P \leq 0.01 ; * * *=P \leq 0.001$

References $(\mathrm{OR}=1)$ respectively: a female, b rural, c occupational group II

Meaning of OR: for example for the missing teeth in all regions: every additional year gives a 3.3\% higher chance for missing teeth. Patients of Occupational group I have 34\% lower chance for missing teeth

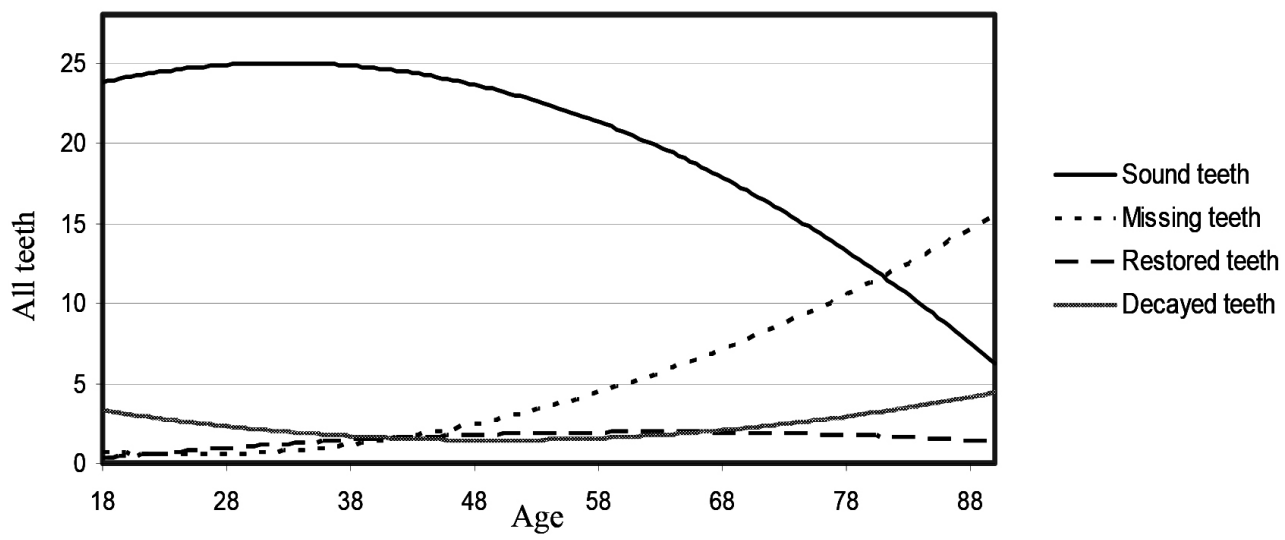

Figure 1. The mean number of decayed, missing and restored teeth in all dental regions with age in 1549 patients before treatment

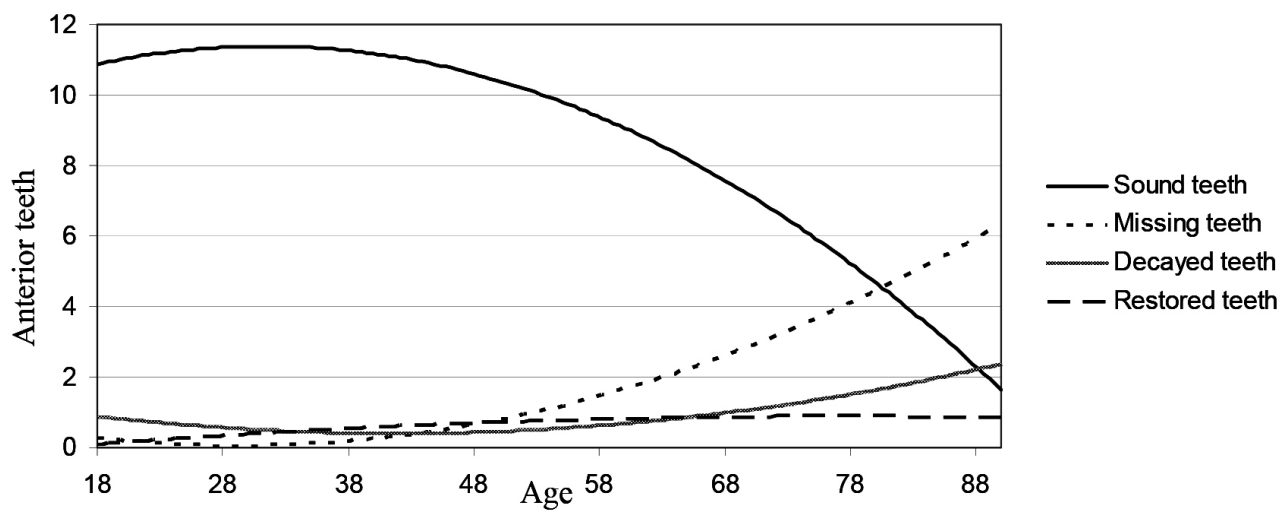

Figure 2. The mean number of decayed, missing and restored teeth in anterior region with age in 1549 patients before treatment 


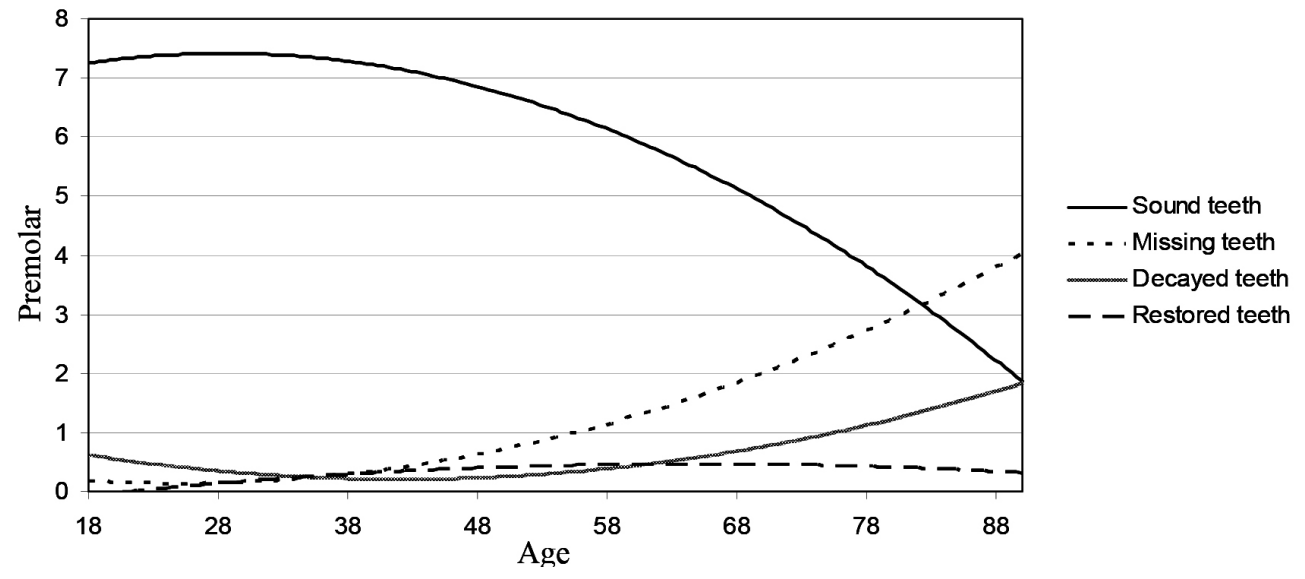

Figure 3. The mean number of decayed, missing and restored teeth in premolar region with age in 1549 patients before treatment

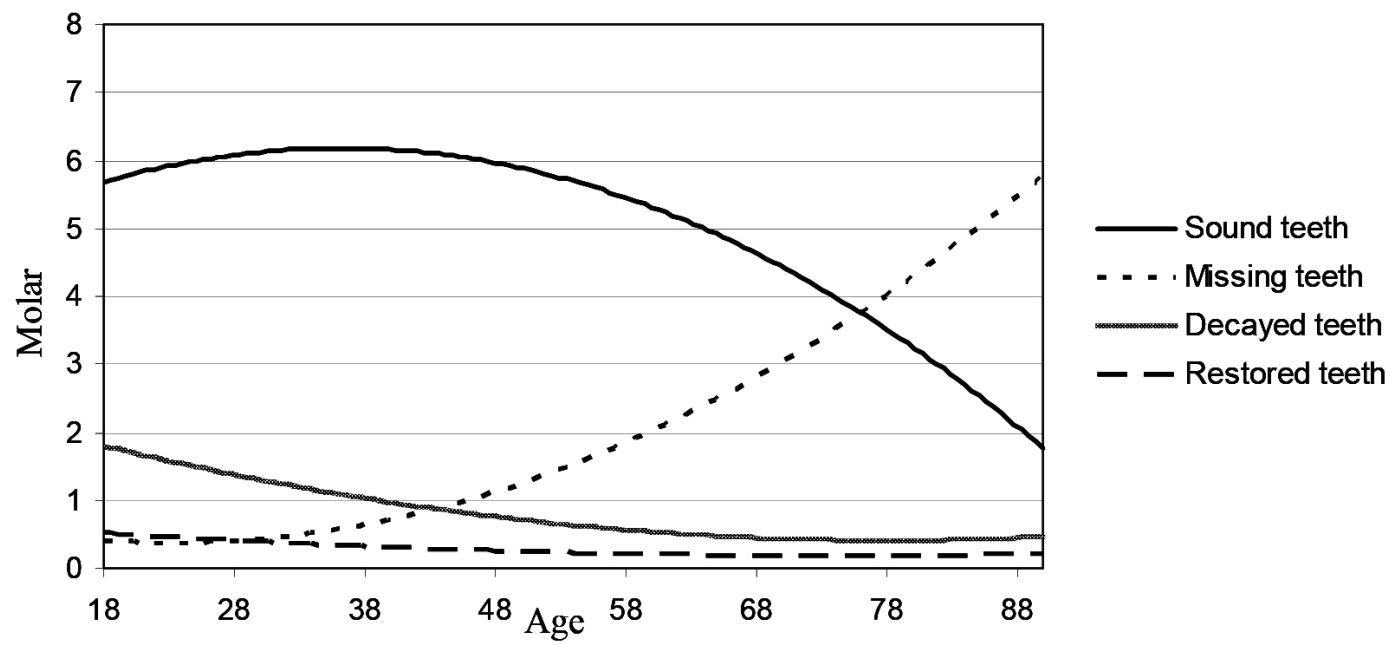

Figure 4. The mean number of decayed, missing and restored teeth in molar region with age in 1549 patients before treatment

to be restored but only 1390 teeth received the fillings and 436 missing teeth were indicated to be replaced but only 363 teeth were replaced.

Restoration was the most common treatment (1390 teeth, 70\%), this was followed by tooth replacement (363 teeth, 18\%) and tooth extraction (219 teeth, 12\%) (Table 2). In the whole dentition, molar teeth were the most common teeth receiving the treatments $(842$ teeth, 43\%), anterior teeth came next (729 teeth, 37\%) and premolar teeth showed the smallest proportion (401 teeth, 20\%) (Table 2 ). In all dental regions, upper anterior teeth and lower molars were the most common teeth receiving the treatments (630 teeth, 32\%; and 546 teeth, $28 \%$ respectively) whilst lower anterior teeth had the smallest proportion of receiving the treatments (99 teeth, 5\%). In all dental regions, the chance for restoration was significantly higher than extraction and teeth replacement (Friedman test $p \leq 0.001$ and Wilcoxon signed ranks test $p \leq 0.017$ ) (Table 3 ). 
Table 2. Distribution of 3600 teeth indicated for treatments and 1972 teeth receiving the treatments in anterior, premolar, and molar regions in the upper jaw, lower jaw, and in the whole dentition according to types of treatment.

\begin{tabular}{|c|c|c|c|c|c|c|c|}
\hline \multirow[t]{3}{*}{ Types of treatment } & \multirow[t]{3}{*}{ Dental region } & \multicolumn{6}{|c|}{ Number of teeth (\%) } \\
\hline & & \multicolumn{2}{|c|}{ Upper jaw } & \multicolumn{2}{|c|}{ Lower jaw } & \multicolumn{2}{|c|}{ Whole dentition } \\
\hline & & Indicated & Treated & Indicated & Treated & Indicated & Treated \\
\hline \multirow[t]{4}{*}{ Tooth extraction } & Anterior & $63(22 \%)$ & $30(26 \%)$ & $20(7 \%)$ & $11(11 \%)$ & $83(15 \%)$ & $41(19 \%)$ \\
\hline & Premolar & $83(29 \%)$ & $34(30 \%)$ & $45(16 \%)$ & $17(16 \%)$ & $128(23 \%)$ & $51(23 \%)$ \\
\hline & Molar & $137(49 \%)$ & $51(44 \%)$ & $212(77 \%)$ & $76(73 \%)$ & $349(62 \%)$ & $127(58 \%)$ \\
\hline & Total & $283(100 \%)$ & $115(100 \%)$ & $277(100 \%)$ & $104(100 \%)$ & $560(100 \%)$ & $219(100 \%)$ \\
\hline \multirow[t]{4}{*}{ Restoration } & Anterior & $746(46 \%)$ & $476(58 \%)$ & $65(7 \%)$ & $39(7 \%)$ & $811(31 \%)$ & $515(37 \%)$ \\
\hline & Premolar & $297(18 \%)$ & $148(18 \%)$ & $181(18 \%)$ & $106(19 \%)$ & $478(18 \%)$ & $254(18 \%)$ \\
\hline & Molar & $577(36 \%)$ & $200(24 \%)$ & $738(75 \%)$ & $421(74 \%)$ & $1315(51 \%)$ & $621(45 \%)$ \\
\hline & Total & $1620(100 \%)$ & $824(100 \%)$ & $984(100 \%)$ & $566(100 \%)$ & $2604(100 \%)$ & $1390(100 \%)$ \\
\hline \multirow[t]{4}{*}{ Tooth replacement } & Anterior & $148(53 \%)$ & $124(56 \%)$ & $55(34.5 \%)$ & $49(35 \%)$ & $203(46 \%)$ & $173(46 \%)$ \\
\hline & Premolar & $70(25 \%)$ & $54(24 \%)$ & $49(31 \%)$ & $42(30 \%)$ & $119(27 \%)$ & $96(28 \%)$ \\
\hline & Molar & $59(22 \%)$ & $45(20 \%)$ & $55(34.5 \%)$ & $49(35 \%)$ & $114(27 \%)$ & $94(26 \%)$ \\
\hline & Total & $277(100 \%)$ & $223(100 \%)$ & $159(100 \%)$ & $140(100 \%)$ & $436(100 \%)$ & $363(100 \%)$ \\
\hline Grand total & & 2180 & 1162 & 1420 & 810 & 3600 & 1972 \\
\hline
\end{tabular}

Table 3. The chance between types of treatment (extraction, restoration, tooth replacement) in dental regions (anterior, premolar, molar) in the upper jaw, lower jaw, and the whole dentition

\begin{tabular}{|c|c|c|c|c|c|c|c|c|}
\hline & \multicolumn{2}{|c|}{ Anterior region } & \multicolumn{2}{|c|}{ Premolar region } & \multicolumn{2}{|c|}{ Molar region } & \multicolumn{2}{|c|}{ All dental region } \\
\hline & $\mathrm{Z}$ & $\mathrm{P}$ & Z & $\mathrm{P}$ & Z & $\mathrm{P}$ & Z & $\mathrm{P}$ \\
\hline \multicolumn{9}{|c|}{ Whole dentition } \\
\hline Ext $^{1}-$ Rest $^{2}$ & $-14.6^{b}$ & $* *$ & $-9.6^{b}$ & $* *$ & $-13.1^{\mathrm{b}}$ & $* *$ & $-19.2^{\mathrm{b}}$ & $* *$ \\
\hline $\operatorname{Repl}^{1}-$ Rest $^{2}$ & $-14.5^{b}$ & $* *$ & $-11.5^{\mathrm{b}}$ & $* *$ & $-18.6^{b}$ & $* *$ & $-24.3^{b}$ & $* *$ \\
\hline $\operatorname{Repl}^{1}-\mathrm{Ext}^{2}$ & $-0.65^{\mathrm{a}}$ & & $-2.6^{\mathrm{b}}$ & $*$ & $-8.08^{b}$ & $* *$ & $-8.2^{\mathrm{b}}$ & $* *$ \\
\hline \multicolumn{9}{|l|}{ Upper jaw } \\
\hline $\mathrm{Ext}^{1}-\mathrm{Rest}^{2}$ & $-14.3^{b}$ & $* *$ & $-7.1^{b}$ & $* *$ & $-7.7^{b}$ & $* *$ & $-16.2^{b}$ & $* *$ \\
\hline $\operatorname{Repl}^{1}-$ Rest $^{2}$ & $-13.9^{b}$ & $* *$ & $-8.5^{b}$ & $* *$ & $-10.9^{b}$ & $* *$ & $-18.9^{\mathrm{b}}$ & $* *$ \\
\hline $\operatorname{Repl}^{1}-\mathrm{Ext}^{2}$ & $-1.3^{\mathrm{a}}$ & & $-1.8^{\mathrm{b}}$ & & $-4.3^{b}$ & $* *$ & $-4.4^{\mathrm{b}}$ & $* *$ \\
\hline \multicolumn{9}{|l|}{ Lower jaw } \\
\hline $\mathrm{Ext}^{1}-\mathrm{Rest}^{2}$ & $-3.3^{b}$ & $* *$ & $-6.8^{b}$ & $* *$ & $-11.9^{\mathrm{b}}$ & $* *$ & $-13.2^{b}$ & $* *$ \\
\hline $\operatorname{Repl}^{1}-\operatorname{Rest}^{2}$ & $-3.07^{b}$ & $*$ & $-7.3^{b}$ & $* *$ & $-15.9^{b}$ & $* *$ & $-17.9^{b}$ & $* *$ \\
\hline $\operatorname{Repl}^{1}-\mathrm{Ext}^{2}$ & $-0.3^{\mathrm{a}}$ & & $-0.6^{\mathrm{b}}$ & & $-5.9^{b}$ & $* *$ & $-7.2^{b}$ & $* *$ \\
\hline
\end{tabular}

$*=p \leq 0.017 ; * *=p \leq 0.001 . \mathrm{a}=$ negative ranks $(2>1) ; \mathrm{b}=$ positive ranks $(1>2)$

Ext: tooth extraction, Rest: restoration, Repl: tooth replacement

Example: Upper anterior teeth significantly have more chance for restoration than tooth extraction

Molars showed statistically significant higher chance for extraction than premolars and anterior teeth (Friedman test $p \leq 0.001$ and Wilcoxon signed ranks test $p \leq 0.017$ ) (Table 4). In the whole dentition and lower jaw, molars had statistically significant higher chance for restoration than premolars and anterior teeth. In the upper jaw, however, anterior teeth had statistically significant highest chance for restoration. The chance for teeth replacement in the upper jaw was greater in the anterior region while in the lower jaw, molars and premolars showed statistically significant higher chance for teeth replacement than anterior teeth. No statistically significance was found in teeth replacement between premolar and molar regions. 
Table 4. The chance of receiving a type of dental treatment (extraction, restoration, tooth replacement) between dental regions (anterior, premolar, and molar) in upper jaw, lower jaw, and in the whole dentition

\begin{tabular}{|c|c|c|c|c|c|c|}
\hline & \multicolumn{2}{|c|}{ Tooth extraction } & \multicolumn{2}{|c|}{ Restoration } & \multicolumn{2}{|c|}{ Tooth replacement } \\
\hline & $\bar{Z}$ & $\mathrm{P}$ & $\mathrm{Z}$ & $\mathrm{P}$ & $\mathrm{Z}$ & $\mathrm{P}$ \\
\hline \multicolumn{7}{|l|}{ Whole dentition } \\
\hline Anterior teeth ${ }^{1}$-Molars ${ }^{2}$ & $-8.2^{\mathrm{b}}$ & $* *$ & $-8.06^{\mathrm{b}}$ & $* *$ & $-2.1^{\mathrm{a}}$ & \\
\hline Anterior teeth ${ }^{1}$-Premolars ${ }^{2}$ & $-3.05^{b}$ & $*$ & $-3.1^{\mathrm{a}}$ & $*$ & $-2.03^{a}$ & \\
\hline Premolars $^{1}$-Molars ${ }^{2}$ & $-6.2^{\mathrm{b}}$ & $* *$ & $-10.5^{b}$ & $* *$ & $-0.4^{\mathrm{a}}$ & \\
\hline \multicolumn{7}{|l|}{ Upper jaw } \\
\hline Anterior teeth ${ }^{1}-$ Molars $^{2}$ & $-4.3^{\mathrm{b}}$ & $* *$ & $-3.6^{\mathrm{a}}$ & $* *$ & $-4.3^{\mathrm{a}}$ & $* *$ \\
\hline Anterior teeth ${ }^{1}$-Premolars ${ }^{2}$ & $-2.6^{\mathrm{b}}$ & $*$ & $-6.8^{\mathrm{a}}$ & $* *$ & $-3.1^{\mathrm{a}}$ & $*$ \\
\hline Premolars $^{1}$-Molars ${ }^{2}$ & $-1.9^{\mathrm{b}}$ & & $-2.5^{\mathrm{b}}$ & $*$ & $-2.1^{\mathrm{a}}$ & \\
\hline \multicolumn{7}{|l|}{ Lower jaw } \\
\hline Anterior teeth ${ }^{1}$-Molars ${ }^{2}$ & $-7.5^{\mathrm{b}}$ & $* *$ & $-15.04^{b}$ & $* *$ & $-2.9^{b}$ & $*$ \\
\hline Anterior teeth ${ }^{1}$-Premolars ${ }^{2}$ & $-1.9^{\mathrm{b}}$ & & $-6.6^{\mathrm{b}}$ & $* *$ & $-2.4^{b}$ & $*$ \\
\hline Premolars $^{1}$-Molars ${ }^{2}$ & $-6.2^{\mathrm{b}}$ & $* *$ & $-11.6^{\mathrm{b}}$ & $* *$ & $-1.7^{\mathrm{b}}$ & \\
\hline
\end{tabular}

$*=p \leq 0.017 ; * *=p \leq 0.001 . \mathrm{a}=$ negative ranks $(2>1) ; \mathrm{b}=$ positive ranks $(1>2)$

Example: Upper molars have higher chance for extraction than upper anterior teeth.

\section{DISCUSSION}

\section{Data collection}

Data collected from the dental records of the patients during a limited period, it could not be considered as representative of the dental patients of CTUMP. However, with a large sample size, the dental treatment strategy drawn from these data could reflect the dental treatment strategy of CTUMP.

Collecting data from dental records have many advantages such as saving time and money, carrying no risk for physical or mental health of participants. ${ }^{22,23}$ However, since databases were only designed for administrative purposes, not to answer any specific research question, the informations that can be helpful for answering a research question may not be available. For example, in this study, in many cases, the information on which teeth indicated to be replaced and on what type of prosthesis (FPD or RPD) indicated were not available in dental records. However, the proportion of patients with teeth replacements in this study was very low (55 cases, 3.6\%).

In addition, the treatment planning and the treatments delivered could be carried out by different dentists, and the discussion between dentists during the treatment process might not be performed. This can introduce the potential bias when the inference on the treatment approach to dental care was drawn from this data. However, majority of the patients had on average two teeth receiving the treatments, this can suggest that the dental treatments received were emergency or in priority in solving problems for their oral function. The informations on the treatment of priority could reflect the treatment approach to dental care.
Oral health status of the patients before treatments The dental patients of CTUMP are part of the population of Southern Vietnam. Hence, the oral health status characteristics of the participants in this study meet the oral health status characteristics of the population in Southern Vietnam as described in an epidemiological study on oral health status of the adults in Southern Vietnam in 2010. ${ }^{18}$ For example, increasing with age, the mean number of missing teeth increases while the mean number of decayed teeth decreases. Patients in professional group 1 have less chance of having missing teeth in all dental regions than the patients in professional group 2 (except for molar region). Men have lower chance of having restored teeth than women in anterior region. The fact that most of them live in the urban areas $(75 \%)$ can explain why no statistically significant on oral health status was found between the urban and rural subjects. Despite of this, urban subjects have lower chance of having decay teeth. The majority of the patients were at young age, most of them have more than 20 teeth $(96 \%)$ that reaches the goal in dental care advocated by WHO (1992). A dentition with at least 20 teeth and 9 or 10 occlusal pairs including the anterior tooth region will satisfy the oral function, masticatory efficiency, occlusal support, and occlusal stability. ${ }^{9,24}$ This may explain why few patients in this study demanded the protheses for teeth replacement (55 cases; 3,6\%).

\section{Dental treatment approach in CTUMP}

On average, one patient only had two teeth receiving the treatments. These treatments can be considered as the solution for the problems in priority of their oral function. Analysing the treatments that aims to solve 
the problems in priority for the oral function can be helpful in determining the treatment approach applied by investigating the dental region that the treatments tend to be focused on.

In dental practice of CTUMP, the chance for teeth replacement in anterior region was higher than in premolar and molar region. This is in line with the findings from a study of Gotfredsen (2007) stating that tooth missing in anterior region is important for most people while tooth missing in molar region is problem for some people, also in line with the finding of a study on the effects of RPD on the quality of life: the patients only benefit RPD when they have tooth missing in anterior region. ${ }^{9,25}$

\section{The dental treatment approach in CTUMP tends to be conservative}

Extraction constituted the low proportion within the dental treatments delivered. Restorations accounted for the high proportion $(1390,70 \%)$ while extraction represented in low percentage, in which majority were root $(219,65 \%$ of extracted teeth) that could not be restored even with the high dental technology available. This is comparable with the findings from the study of Pham Hai Dang (2010) on the treatment needs and treatment demands of the dental patients of CTUMP. ${ }^{26}$ The findings from this study showed that most of dental treatments were restorations, while the extractions were in low percentage. This trend contrasts with the dental treatment status in the hospitals and the health centres where extractions are the most common dental treatment. ${ }^{27}$ The discrepancy in the dental treatment trend between CTUMP and other govermental health services can be explained by the discrepancies in the background of the patients and of the dental resources since the variety of the treatment planning depends on the patients, dentists, treatment approach and dental resources. ${ }^{14,27}$ Most of patients in this study are in middle and high SES (profession group I), live in urban area, at young age - the population group that have higher chance for accessibility to the conservative dental treatments due to the accessibility to the dental care. ${ }^{18}$ At the dentist level, the dental practicians of CTUMP are lecturers who were considered to be well equipped with knowledge and skills in dentistry, with a conservative morphological approach to dental care guiding the dentistry in Vietnam up to now. Regarding the financial resources, different from others govermental health services, CTUMP is well equipped with high dental technologies in order to perform most of conservative dental treatments as possible. In addition, most of dental treatment fees are covered in the budget of CTUMP in order to facilitate the dentists and the patients to access the advanced conservative dental technologies. Hence, the dental treatments are offerred with an affordable cost. Therefore, the accessibility to the conservative dental treatments of the patients in CTUMP is not surprising.

\section{The dental treatment approach in CTUMP tends to be morphologically conservative}

The dental treatments aims to preserve all teeth regardless of dental regions. Most of dental treatment are restorations. Moreover, molar region has higher chance for receiving restoration than for extraction and higher chance for receiving restoration than anterior region and premolar region despite of the fact that molars are high risk teeth for dental caries and periodontal diseases compared to anterior and premolar region, and that the treatments on molars are more complicated with uncertain prognosis and expensive. ${ }^{28}$ Although anterior region has higher chance for teeth replacement, there is no statistical significance found between premolar region and molar region for this event. This implies that teeth replacement in dental practice of CTUMP may tends to be morphological rather than functional by providing the prostheses that restore the complete dental arch regardless of premolars or molars. This is in line with the findings from a survey on prosthodontic production of laboratory in Southern Vietnam. ${ }^{29}$ The finding from that study showed that in Southern Vietnam, "the prosthodontic service aims at morphologic rather than functional tooth replacement, as it tends to provide prostheses leading to 'complete dental arches"'. There are evidences on the benefits and the disadvantages of RPD replacing missing molars. ${ }^{14,25,30}$ In many cases, RPDs in molar region are not only helpless in improving the oral function but also create the discomfort, increase risk of caries and periodontal diseases. That may lead to overtreatment in many cases, especially in case of shortened dental arches, the incomfort of wearing RPD in molar region is the most common reason for discarding the prostheses. Therefore, the patients usually demand tooth replacement in anterior region and premolar region. ${ }^{14,25,31}$ However, since the informations on dental arch types of the patients and teeth to be replaced were not available in many dental records, data from this study do not allow us to drawn a remark on the treatment approach in prosthodontic treatment in CTUMP.

This finding suggests that the problem-solving approach given in the dental curriculum of CTUMP has not been implemented in the oral care. The educational background of the dental teachers of CTUMP may constitute a reason for this. Although the problemsolving approach has been integrated in the dental curriculum, the teachers may not get acquainted with the new approach since most of them have adopted the traditional approach to dental care during their dental undergraduate education. Moreover, there is not a protocol of problem-based treatment planning in dental practice of CTUMP, it supposes that the problemsolving approach to dental care is not systematically practised in CTUMP. 


\section{CONCLUSION}

In conclusion, the approach to dental care in CTUMP tends to be morphologically conservative. The morphologically conservative approach to dental care showed many drawbacks. It should be replaced by the problem-solving-approach. This approach should be integrated not only in the theory of the dental curriculum but also in the daily dental practice of the teachers and the students. Further study on the approach to dental care by investigating treatment demands of the patients and treatment planning in dental practice of CTUMP should be conducted.

\section{REFERENCES}

1. Kayser AF. Shortened dental arch: a therapeutic concept in reduced dentitions and certain high-risk groups. Int J Perio Restor Dent. 1989;9:426-49.

2. Broughton AM, Smales RJ. Comparison of dental needs with the treatments actually received. Aust Dent J. 1991;36:223-30.

3. Käyser AF. How much reduction of the dental arch is functionally acceptable for the ageing patient? Int Dent J. 1990;40:183-8.

4. Elderton RJ. Overtreatment with restorative dentistry: when to intervene? Int Dent J. 1993;43:1724.

5. Dawson AS, Makinson OF. Dental treatment and dental health. Part 2. An alternative philosophy and some new treatment modalities in operative dentistry. Aus Dent J. 1992;37:205-10.

6. De Sa e Frias V, Toothaker R, Wright RF. Shortened dental arch: a review of current treatment concepts. J Prosthodont. 2004;13:104-10.

7. Ramfjord SP, Ash MM. Occlusion. 3rd ed. Philadelphia: Saunders, 1983.

8. Mohl ND, Zarb GA, Carlsson GE, Rugh JD. A textbook of occlusion. Chicago: Quintessence, 1988.

9. Gotfredsen K, Walls AW. What dentition assures oral function? Clin Oral Implants Res. 2007;18(Suppl 3):34-45.

10. Käyser AF. Limited treatment goals - shortened dental arches. Periodontol 2000. 1994;4:7-14.

11. Witter DJ, Creugers NHJ, Kreulen CM, de Haan AF. Occlusal stability in shortened dental arches. J Dent Res. 2001;80:432-6.

12. Ciancaglini R, Gherlone EF, Radaelli G. Association between loss of occlusal support and symptoms of functional disturbances of the masticatory system. J Oral Rehabil. 1999;26: 24853.
13. Tallents RH, Macher DJ, Kyrkanides S, Katzberg RW, Moss ME. Prevalence of missing posterior teeth and intraarticular temporomandibular disorders. J Prosthet Dent. 2002;87:45-50.

14. Kanno T, Carlsson GE. A review of the shortened dental arch concept focusing on the work by the Käyser/Nijmegen group. J Oral Rehabil. 2006;33:850-62.

15. Steele JG, Ayatollahi SMT, Walls AWG, Murray JJ. Clinical factors related to reported satisfaction with oral function amongst older adults in England. Community Dent Oral Epidemiol. 1997;25:143 -9.

16.. Türp JC, Greene CS, Strub JR. Dental occlusion: a critical reflection on past, present and future concepts. J Oral Rehabil. 2008;35:446-53.

17. Do LG, Spencer AJ, Roberts-Thomson K, Trinh HD, Nguyen TT. Oral health status of Vietnamese adults: Findings from the National Oral Health Survey of Vietnam. Asia Pac J Public Health. 2011;23:228-36.

18. Nguyen TC, Witter DJ, Bronkhorst EM, Truong NB, Creugers NH. Oral health status of adults in Southern Vietnam - a cross-sectional epidemiological study. BMC Oral Health. 2010;13,10:2.

19. Statistical data. General office for population family planning. Distribution of land area and population by region [Internet]. [cited 2009 Sept 17]. Available from: http://www.e.gopfp.gov.vn/ web/guest/data.

20. Khanh ND. Report of dental care in Southern Vietnam 2008. Southern Vietnam Dental Congress XI 2008.

21. Statistical data. World Health Organization. Statistical tables 2009. Regional office of the Western Pacific 2009 [Internet]. [cited 2011 Jan]. Available from: http://www.who.int.

22. Patricia Huston, C. David Naylor, Dphil. Health services research: reporting on studies using secondary data sources. Canadian Medical Association. 1996;155:1697-709.

23. Sarah Boslaugh. Secondary Data Sources for Public Health: A Practical Guide. Cambridge University Press. 2007 Apr 9.

24. Elias AC, Sheiham A. The relationship between satisfaction with mouth and number and position of teeth. J Oral Rehabil. 1998;25:649-61.

25. Armellini DB, Heydecke G, Witter DJ, Creugers NHJ. Effect of removable partial dentures on oraol health-related quality of life in subjects with shortened dental arches: a 2-center cross-sectional study. Int J Prosthodont. 2008;21:524-30. 
26. Pham Hai Dang. Treatment needs and treatment demands of patients of Cantho university of medicine and pharmacy and patients of Cantho hospital [dissertation]. Cantho university of medicine and pharmacy; 2010.

27. Van Palenstein Helderman W, Mikx F, Truin GJ, Hoang TH, Pham HL. Workforce requirements for a primary oral health care system. Int Dent J. 2000;50:371-7.

28. Witter DJ, van Palenstein Helderman WH, Creugers NHJ, Kayser AF. The shortened dental arch concept and its implications for oral health care. Community Dent Oral Epidemiol. 1999;27:249-58.
29. Chau TN, Witter DJ, Kreulen CM, Creugers NH, Tu HH. Replacement of missing teeth in a southern region of Vietnam-a descriptive dental laboratory study. Int Dent J. 2009;59:192-6.

30. Witter DJ, Van Elteren P, Käyser AF, Van Rossum GM. Oral comfort in shortened dental arches. J Oral Rehabil. 1990;17:137-43.

31. Meeuwissen JH, van Waas MA, Meeuwissen R, Käyser AF, van 't Hof MA, Kalk W. Satisfaction with reduced dentitions in elderly people. J Oral Rehabil. 1995;22:397-401.

(Received November 11, 2014; Accepted February 15, 2015) 